\title{
Real Time Speed Bump Detection Using Gaussian Filtering and Connected Component Approach
}

\author{
W. Devapriya ${ }^{1}$, C. Nelson Kennedy Babu ${ }^{2}$, T. Srihari ${ }^{3}$ \\ ${ }^{1}$ Electronics and Communication Engineering, KSR Institute for Engineering and Technology, Tiruchengode, \\ Namakkal, India \\ ${ }^{2}$ Computer Science Engineering, Dhanalakshmi Srinivasan College of Engineering, Coimbatore, India \\ ${ }^{3}$ Electrical and Electronics Engineering, KSR Institute for Engineering and Technology, Tiruchengode, Namakkal, \\ India \\ Email:w.devapriyya@gmail.com,cnkbabu63@gmail.com, k.t.srihari@gmail.com
}

Received 30 March 2016; accepted 20 April 2016; published 12 July 2016

Copyright (C) 2016 by authors and Scientific Research Publishing Inc.

This work is licensed under the Creative Commons Attribution International License (CC BY). http://creativecommons.org/licenses/by/4.0/

\section{(c) (i) Open Access}

\section{Abstract}

An Intelligent Transportation System (ITS) is a new system developed for the betterment of user in traffic and transport management domain area for smart and safe driving. ITS subsystems are Emergency vehicle notification systems, Automatic road enforcement, Collision avoidance systems, Automatic parking, Map database management, etc. Advance Driver Assists System (ADAS) belongs to ITS which provides alert or warning or information to the user during driving. The proposed method uses Gaussian filtering and Median filtering to remove noise in the image. Subsequently image subtraction is achieved by subtracting Median filtered image from Gaussian filtered image. The resultant image is converted to binary image and the regions are analyzed using connected component approach. The prior work on speed bump detection is achieved using sensors which are failed to detect speed bumps that are constructed with small height and the detection rate is affected due to erroneous identification. And the smartphone and accelerometer methodologies are not perfectly suitable for real time scenario due to GPS error, network overload, real-time delay, accuracy and battery running out. The proposed system goes very well for the roads which are constructed with proper painting irrespective of their dimension.

\section{Keywords}

Intelligent Transportation System, Speed Bumps, Driver Assistance System, Gaussian and Median Filtering, Connected Component Analysis 


\section{Introduction}

Driver assistance system is an important module in Intelligent Transportation System (ITS). The system is developed to alert a driver or to interact directly on the vehicle for safety and better driving. DAS includes Driver drowsiness detection, Adaptive cruise control (ACC), Lane departure warning system, Traffic sign recognition, Wrong-way driving warning, automotive navigation system, etc. In addition to that here we focused on obstacle detection in road side like speed bump, poth holes, etc.

Speed bumps are constructed across the road to avoid over speed in restricted area. The most critical thing is preventing remedy leading to the cause of accident. Because many speed bumps are constructed without proper permission. Un-notification of speed bump over high speed is harmful for patients in transit, pregnant women, rapid wear and tear and damage to vehicles. So we develop a system that services the end user driver using image processing concepts-Gaussian filtering, Median filtering and Connected Component Approach. This paper is organized as follows: Chapter 2 describes the background and related works and Chapter 3 refers data collection. The proposed methodology is covered in Chapter 4 and result and discussion are covered in Chapter 5 . Finally conclusion and future scope are explained in Chapter 6.

\section{Background and Related Works}

The earlier approach of speed bump detection is achieved using dedicated sensors, three-axis accelerometer, Smart Phone and Image Processing.

Using Sensors: Hull et al. developed a distributed mobile sensor computing system, [1]. The system built with set of sensors embedded in vehicles to collect and process data and send it to portal based upon the continuous queries which are processed by continuous query processor on remote nodes. In paper [2] [3] real-time free space detection system is implemented using a medium-cost LIDAR sensor and a low cost camera. In paper [4] [5] the speed bump detection is done with bump recorder, pedometer, three-dimensional gyro sensor and GPS. The drawback of using sensor is miss classification of speed bump.

Smartphone: Nericell [6] used mobile Smartphone to monitor road and traffic conditions. It detected potholes, braking, bumps and honks using accelerometer, microphone, GSM radio and GPS sensors inbuilt in smart phones. One constraint is the phone must be oriented along the vehicle's axis before analyzing the signals. Patrol system [7] uses 3-axis accelerometer and GPS mounted on the dashboard to monitor road surface. Wolverine [8] method uses Smartphone sensors for traffic state monitoring and detection of bumps without orientation constrain. They give 10\% false negative rate for bump detection. The work carried out in paper [9] [10] is using a mobile smartphone, they demonstrated an applications that are integrated inside an automobile to evaluate a vehicle's condition, such as gear shifts and overall road conditions, including bumps, potholes, rough road, uneven road, and smooth road. The mobile smartphone holds GPS, microphones, and a Bosch BMA150 3-axis accelerometer. And also in paper [11]-[13] they implemented an early warning system that uses a smart phone and accelerometer to alert the driver in advance when the vehicle is approaching a speed breaker. Gunjan Chugh [9] gave a summarized paper on road condition detection using dedicated sensors and smart phones. In the same paper they highlighted the disadvantage of using smart phones and GPS for speed bump. The drawbacks of the using smartphone system are vibration patterns of sensor data, benign events, GPS error, network overload, delay and battery draining. One of the most common methodology for speed bump detection is using smartphone, the problem arises because of its hard code nature. It is so called, since the detection of speed bump is based on the previous history not based on current scenario so it is unfit for real time scenario.

Image processing: In paper [14] they proposed a methodology to detect speed bump using Disparity, Border detection, Morphological Image processing, canny edge detector concepts. A simple edge detection methodology can't be suitable to detect speed bump in real-time. K. Ganesan [15] proposed an image processing approach to detect obstacles on road using a monocular IR camera. Their main focus is to compensate shadows in the road Using Open Source Computer Vision (OpenCV). In the work on paper [16] the speed bump are detected using morphological and projection analysis. Compare to the previous work on image processing accuracy rate is improved for all category of speed bump and this new methodology also suitable for non-marking speed bump.

The developed system is applicable for trained and untrained routes whereas the smartphone method is applicable only for trained routes. 


\section{Experimental Setup}

The image is captured by locating the camera in front of the vehicle. Camera location and orientation outside the car should be aligned in such a way that focuses on the roads to capture the speed bump image. The database consists of nearly 1500 image including all category. The category is grouped based on the pattern, size and width of the speed bump. All the data collected by the camera are stored on the memory and processed. A 5 MB pixel camera along with a Raspberry Pi is involved in this system. Raspberry is the processing unit interfaced with camera and alerting system. In the proposed work, video is captured via camera and the frames are converted into image files under the hand of converting unit. In the next stage the proposed methodology computation are executed. And finally based on speed bump detection the driver is alerted either by means of alarm or warning indicator. Figure 1 shows the experimental setup flowchart.

\section{Proposed Methodology}

The proposed methodology involves 4 stages as shown in Figure 2. The first stage is preprocessing which prepare the input image before doing the actual process. For the resultant image we apply Gaussian and Median filtering followed by image subtraction. The ensuing subtracted image is converted to binary image and finally from connected component approach speed bump parameter are computed.

\subsection{Pre-Processing}

Pre-processing is an important procedure which helps to remove the unwanted information like noise and strengthens the required information in an image. In projected method the preprocessing involve 1) Resize to standard size 2) RGB to Gray scale conversion [17].

\subsubsection{Resize to Standard Size}

Resize is mandatory to reduce the computational complexity. All input image are resized to $140 \times 320$ size. The focus is to detect the presence speed bump so we go for granular analysis. The original image is shown in Figure 3.

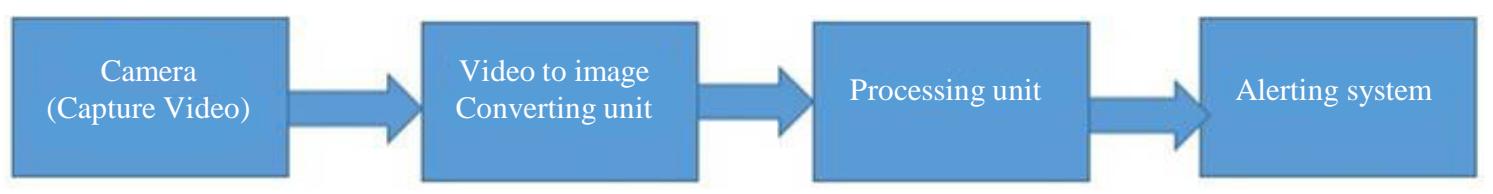

Figure 1. Experimental setup flowchart.

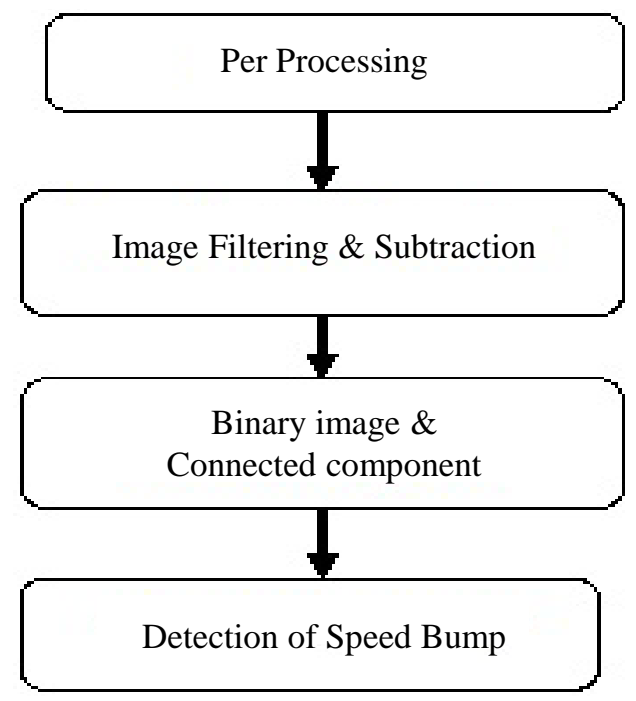

Figure 2. Proposed method flowchart. 


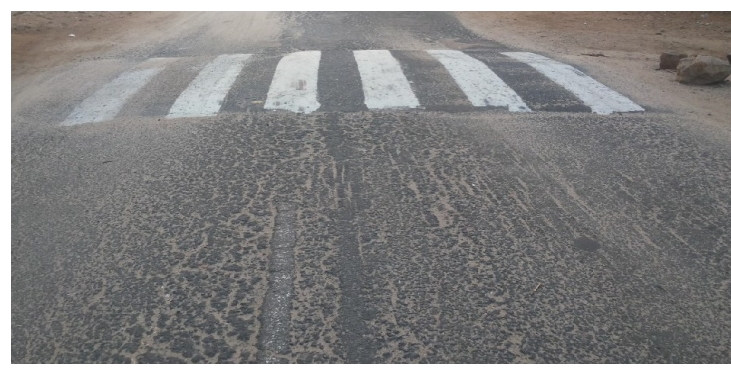

Figure 3. Sample RGB color image.

\subsubsection{Convert RGB to Gray Scale Image}

An RGB image is altered to gray scale image [18] by using (1). The corresponding gray scale image is displayed in Figure 4. Human eyes are more sensitive to green color than red and blue color so green is assumed high value and the formula for computing luminosity is given by

$$
L(x, y)=0.21 R(x, y)+0.72 G(x, y)+0.07 B(x, y)
$$

where

$\mathrm{R}$-Red component of the image;

$\mathrm{G}$ - Green component of the image;

$\mathrm{B}$-Blue component of the image.

\subsection{Image Filtering and Subtraction}

\subsubsection{Apply Gaussian Filtering}

The significance of a low pass Gaussian filtering is to remove noise by blurring the image and remove the high frequency component of the image. Here the environmental noise like sand particle in the road, uneven road conditions are eliminated by the influence of Gaussian filtering. The degree of smoothness depends on the value of standard deviation and kernel size chosen. (2) refers the Gaussian filtering where $\sigma$ refers the standard deviation, $\sigma^{2}=$ variance. In Gaussian curve [19] more weights are at the center and reduced towards the end which distinguish it from the other filter like mean filters (uniformly weighted)

$$
g(x, y)=\frac{1}{2 \pi \sigma^{2}} e^{-\left(\frac{x^{2}+y^{2}}{2 \sigma^{2}}\right)}
$$

We assume the standard deviation 2 and kernel function of size 49. The kernel size is assumed higher value since the focus are on granular not on fine image according to the application. In speed bump detection we are not in need of detailed information. The Gaussian filter output at this stage is shown in the Figure 5.

\subsubsection{Median Filtering}

Median filtering is a nonlinear filter which are good in reducing impulsive noise but the specialty is they safeguard the edges in an image as opposite to linear smoothing filters [20]. For the Gaussian output we apply median filtering of size $31 \times 31$. Among the 961 value it choose the median value, the size of the filter is assumed high for easy computation and at the same time we ensure to maintain the required information. The output at this stage is as shown in Figure 6.

\subsubsection{Image Subtraction}

Subtracting the median filter output from Gaussian filtering output highlight the edge variation [17]. The resulting output is added with a number 127 to perform Binary image. Figure 7 displays the subtracted image.

\subsection{Binary Image and Connected Component}

\subsubsection{Covert to Binary Image}

The subtracted image is converted to binary image by a simple technique named thresholding [17]. Deciding 


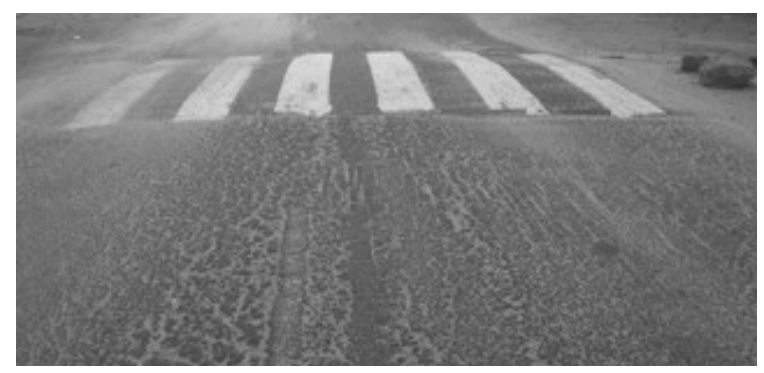

Figure 4. Gray scale image.

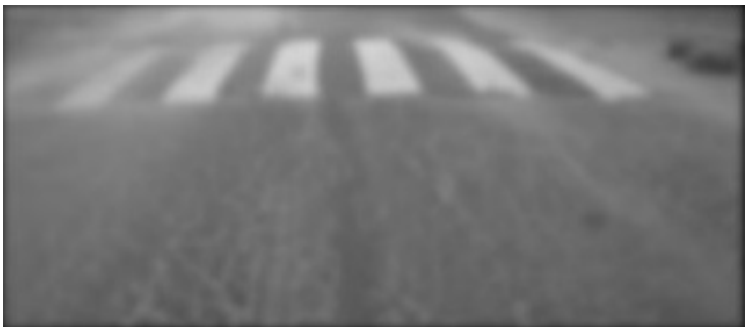

Figure 5. Gaussian filter output.

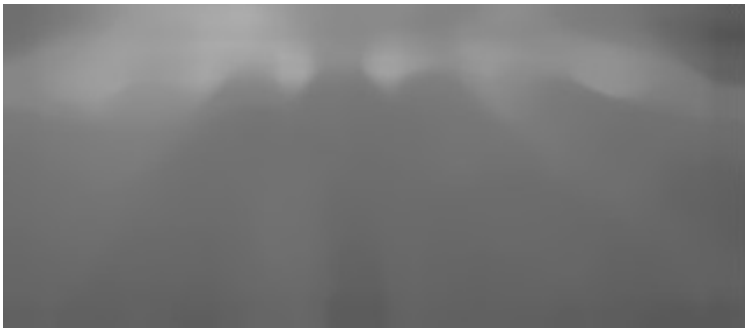

Figure 6. Median filter output.

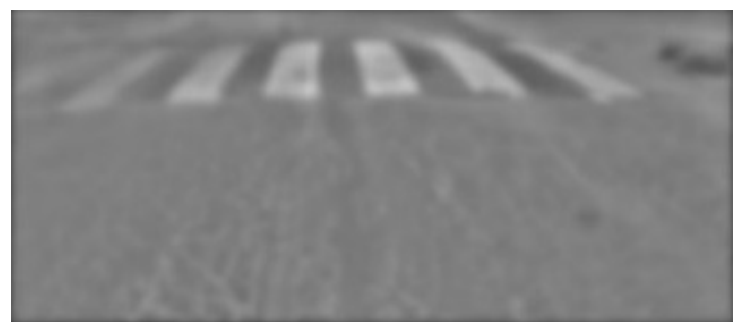

Figure 7. Subtracted results.

threshold value is usually a tough job, but in our work it is very simple because of the nature of application. At the end of this stage the image are seen as binary image with highlighting the white region and removing the unwanted region as exposed in Figure 8. By viewing the result, there is a possibility of highlight some noise. To remove such noise we move on to next stage called connected component approach.

\subsubsection{Analysis Connected Component}

At this stage we apply area open operation of connected component method to stay back with area above a threshold value and remove other noisy region [18]. The threshold level is set nominal such that it contains only the white pattern on the speed bump. The output of this process is revealed in Figure 9.

\subsection{Predict Speed Bump}

The resulting output pattern can be trained using neural network to recognize the speed bump and alert the 


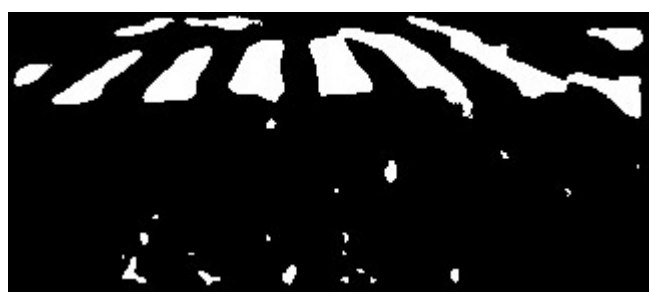

Figure 8 . Binary image result.

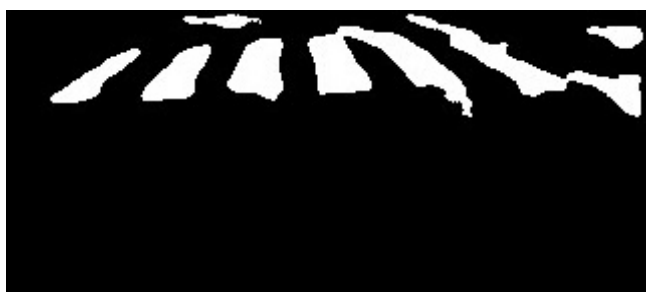

Figure 9. Output of opening operation.

Table 1. Result of true positive and true negative percentage category wise.

\begin{tabular}{cccc}
\hline Category & No. of Sample & True Positive & True Negative \\
\hline Category 1 & 500 & $92 \%$ & $\mathbf{8 \%}$ \\
Category 2 & 300 & $90 \%$ & $10 \%$ \\
Category 3 & 300 & $89 \%$ & $11 \%$ \\
Category 4 & 200 & $90 \%$ & $10 \%$ \\
Category 5 & 200 & $30 \%$ & $70 \%$ \\
\hline
\end{tabular}

Table 2. Resultant output of proposed method for different categories.

Category


driver by means of an audio signal or reducing the speed of the vehicle automatically.

\section{Result and Discussion}

In India it is not an easy task to categories the speed bumps due to lots of variation in the construction. The variation is seen in terms of their pattern, color, length, width, and height. For the analysis we categories the speed bump only based on color and pattern irrespective to their dimensionality. To determine the performance of the system 2 parameter are consider namely True Positive, True Negative. True Positive means identifying the Presence of Speed bump as Presence whereas True Negative implies the Presence of Speed bump as Absence.

Category 1 is the most common type consists of 500 samples in which 460 sample are identified correctly as speed bump the remaining 40 are not recognized since the marking is below the threshold level that happens due to fading and noise. Table 1 shows the True Positive and True Negative Percentage for each category. From the analysis, we concluded that the category 1, 2, 3 and 4 achieve nearly 90\% detection rate on Speed bump detection except category 5 which obtain only less percentage. This infers that the speed bump with marking can be easy identified compare to unmarking speed bump. The performance of the system is reduced due to the fading and unwanted noise on the marking speed bump. Thus the proposed methodology suits very well for the road with proper condition. Even though category 5 does not have marking over it the detection of speed bump happens since the top layer of speed bump is above the threshold value. Table 2 contains a sample collection of input image for each category and its corresponding processed output. The result clearly shows that speed bumps constructed with proper marking are detected easily irrespective of unsmooth road condition and even when the marking is not perfect.

\section{Conclusion and Future Scope}

In this paper, we have proposed a novel method of speed bump detection using Gaussian, median filtering, image subtraction, binary image conversion and connected component approach concepts that alert the driver during his driving. In particular this methodology suits very well to the real time scenario for the painted speed bump though their pattern, color and dimensionality and road condition varies. In addition it partially identifies illegal speed bumps (speed bumps that falls under category 5). This methodology is robust and effortless to implement in standalone machine that avoids congestion in networking (GPS), saving battery of smartphones while driving. The future scope of the proposed work is detection of bumps in night vision and bad illumination condition like raining and mist.

\section{References}

[1] Bychkovsky, V., Chen, K., Goraczko, H., Hu, H., Hull, B., Miu, A., Shih, E., Zhang, Y., Madden S. and Balakrishnan, H. (2006) The Cartel: A Distributed Mobile Sensor Computing System. 4th International Conference on Embedded Networked Sensor Systems, Boulder, November 2006, 125-138. http://dx.doi.org/10.1145/1182807.1182866

[2] Fernández, C., et al. (2012) Free Space and Speed Humps Detection Using Lidar and Vision for Urban Autonomous Navigation. 2012 IEEE Intelligent Vehicles Symposium (IV), Alcala de Henares, 3-7 June 2012, 698-703. http://dx.doi.org/10.1109/IVS.2012.6232255

[3] Zhang, W. (2010) LIDAR-Based Road and Road-Edge Detection. 2010 IEEE Intelligent Vehicles Symposium (IV), San Diego, 21-24 June 2010, 845-848. http://dx.doi.org/10.1109/IVS.2010.5548134

[4] Yagi, K. (2010) Extensional Smartphone Probe for Road Bump Detection. 17th ITS World Congress, Busan, 25-29 October 2010.

[5] Goregaonkar, R.K. and Bhosale, S. (2014) Assistance to Driver and Monitoring the Accidents on Road by Using Three Axis Accelerometer and GPS System. International Journal of Electronics Communication and Computer Engineering, 5, Technovision-2014.

[6] Mohan, P., Padmanabhan, V.N. and Ramjee, R. (2008) Nericell: Rich Monitoring of Road and Traffic Conditions Using Mobile Smartphones. Proceedings of the 6th ACM conference on Embedded Network Sensor Systems, New York, 323-336. http://dx.doi.org/10.1145/1460412.1460444

[7] Eriksson, J., Girod, L., Hull, B., Newton, R., Madden, S. and Balakrishnan, H. (2008) The Pothole Patrol: Using a Mobile Sensor Network for Road Surface Monitoring. 6th Annual International Conference on Mobile Systems, Applications and Services (MobiSys 2008), Breckenridge, 17-20 June 2008, 29-39. http://dx.doi.org/10.1145/1378600.1378605

[8] Bhoraskar, R., Vankadhara, N., Raman, B. and Kulkarni, P. (2012) Wolverine: Traffic and Road Condition Estimation 
Using Smartphone Sensors. 2012 Fourth International Conference on Communication Systems and Networks (COMSNETS), Bangalore, 3-7 January 2012, 1-6. http://dx.doi.org/10.1109/COMSNETS.2012.6151382

[9] Chugh, G., Bansal, D. and Sofat, S. (2014) Road Condition Detection Using Smartphone Sensors: A Survey. International Journal of Electronic and Electrical Engineering, 7, 595-601.

[10] Fazeen, M., Gozick, B., Dantu, R., Bhukhiya, M. and González, M.C. (2012) Safe Driving Using Mobile Phones. IEEE Transactions on Intelligent Transportation Systems, 13, 1462-1468. http://dx.doi.org/10.1109/TITS.2012.2187640

[11] Jain, M., et al. (2012) Speed-Breaker Early Warning System. NSDR, Boston, 15 June 2012.

[12] Tonde, V.P., et al. (2015) Road Quality and Ghats Complexity Analysis Using Android Sensors. International Journal of Advanced Research in Computer and Communication Engineering, 4.

[13] Mahajan, D.V. and Dange, T. (2015) Analysis of Road Smoothness Based on Smartphones. International Journal of Innovative Research in Computer and Communication Engineering, 3.

[14] Bahena, J.M.R., et al. (2010) Speed Booms Detection for a Ground Vehicle with Computer Vision. Proceedings of the 12th WSEAS International Conference on Mathematical and Computational Methods in Science and Engineering. 258-264.

[15] Ganesan, R.K., et al. (2015) An Image Processing Approach to Detect Obstacles on Road. SAE Technical Paper, No. 2015-26-0006.

[16] Devapriya, W., Nelson Kennedy Babu, C. and Srihari, T. (2015) Advance Driver Assistance System (ADAS)—Speed Bump Detection. 2015 IEEE International Conference on Computational Intelligence and Computing Research, Madurai, 10-12 December 2015, 1-6. http://dx.doi.org/10.1109/ICCIC.2015.7435753

[17] Gonzalez, C. and Woods, R.E. (2013) Digital Image Processing, Person. 3rd Edition, Person, New Delhi, $635,738$.

[18] Devapriya, W., Babu, C. and Srihari, T. (2015) Indian License Plate Detection and Recognition Using Morphological Operation and Template Matching. World Academy of Science, Engineering and Technology, International Science Index 100. International Journal of Computer, Electrical, Automation, Control and Information Engineering, 9, 10121018.

[19] Jameel, S.A. and Mohamed Shanavas, A.R. (2015) Implementation of Improved Gaussian Filter Algorithm for Retinal Fundus Images. International Journal of Computer Applications, 132, 1-4. http://dx.doi.org/10.5120/ijca2015907489

[20] Ali Ahmed, E.S., Elatif, R.E.A. and Alse, Z.T. (2015) Median Filter Performance Based on Different Window Sizes for Salt and Pepper Noise Removal in Gray and RGB Images. International Journal of Signal Processing, Image Processing and Pattern Recognition, 8, 343-352. http://dx.doi.org/10.14257/ijsip.2015.8.10.34

\section{Submit or recommend next manuscript to SCIRP and we will provide best service for you:}

Accepting pre-submission inquiries through Email, Facebook, Linkedin, Twitter, etc

A wide selection of journals (inclusive of 9 subjects, more than 200 journals)

Providing a 24-hour high-quality service

User-friendly online submission system

Fair and swift peer-review system

Efficient typesetting and proofreading procedure

Display of the result of downloads and visits, as well as the number of cited articles

Maximum dissemination of your research work

Submit your manuscript at: http://papersubmission.scirp.org/ 